\title{
Taponamiento cardíaco como forma de presentación de un tumor de origen desconocido: abuso en las pruebas diagnósticas
}

\author{
D. Soto de Prado, I. Martín, A. Ocaña, I. Ruiz, J. del Valle, C. Delgado, L. Bellido, M. Navarro, E. Soto, \\ E. Ceballos, G. Martín, J. J. Cruz
}

\section{Resumen}

- Proposito: Presentamos el caso de un tumor de origen desconocido que debutó como derrame pericardio con taponamiento cardiaco. El estudio necrópsico del pericardio resultó ser adenocarcinoma pobremente diferenciado de pulmón.

- Material y métodos: Mujer de 60 años que presento cuadro de taponamiento cardiaco secundario a un derrame pericárdico metastásico de un tumor no filiado tras las pertinentes pruebas diagnósticas.

- Resultados y conclusiones: Los tumores de origen desconocido (TOD) son un problema relativamente frecuente en la práctica clínica oncológica, estimándose su incidencia en un 5-10\% de todos los enfermos con cáncer ${ }^{1}$, presentando un problema de dificultad diagnóstica y manejo terapéutico. La forma de presentación clínica varía dependiendo de la localización a distancia sin existir un patrón metastásico similar al de sus homólogos con primario establecido. Así mismo la búsqueda exhaustiva del tumor primario suele ser la mayoría de las veces improductiva y muy costosa ${ }^{2}$.

\section{Palabras clave:}

Tumor de origen desconocido. Taponamiento cardiaco. Rentabilidad de las pruebas diagnósticas.

Oncología, 2005; 28 (6):293-296

Servicio de Oncología Médica

Hospital Clínico Universitario

Salamanca 


\section{Summary}

- Purpose: We report the case of a woman with cancer of unknown primary site, presented with pericardial effusion and cardiac tamponade. Necropsy showed a pericardium with poorly differentiated lung adenocarcinoma lesions.

- Material and Methods: We present a 60 year old woman who after the pertinent tests was diagnosed of cardiac tamponade secondary to pericardial metastasis of cancer of unknown primary site.

- Results and conclusions: Cancer of unknown primary site is a very common problem in the oncology clinical practice, that has been estimated between 5 and $10 \%$ of all cancers, representing a common medical problem because of the difficult diagnosis and therapeutic management. The clinical manifestations depend on the localization of the distant metastases, whose pattern differs from that of the metastatic localizations of the homologous cancers of known primary site. The lack of results after an exhaustive search make further studies unnecessary.

Key words: Cancer of unknown primary site. Cardiac tamponade. Profitability of diagnostic tests.

\section{Introducción}

Los tumores de origen desconocido son una entidad relativamente frecuente, con una incidencia estimada en un 5-10\% de todos los enfermos de cáncer $^{1}$. Comprende un grupo heterogéneo de enfermedades neoplásicas que se presentan con metástasis sin identificación del tumor primario, con un pronóstico malo y una mediana de supervivencia de 610 meses $^{3,4}$ a pesar de las mejoras en el tratamiento quimioterápico y los progresos en el diagnóstico patológico. Presentamos el caso de una mujer a la que se le diagnostico de tumor de origen desconocido que debutó como derrame pericardio con taponamiento cardiaco y cuyo estudio necrópsico del pericardio fue compatible con adenocarcinoma pobremente diferenciado de pulmón.

\section{Caso clínico}

Presentamos el caso de una mujer de 60 años, con antecedentes de osteoporosis en tratamiento con Bifosfonatos semanal que acude al Servicio de Urgencias por cuadro de plenitud gástrica postpandrial, dispepsia y nauseas de 4 días de evolución además de dolor en región costal derecha desde hace un mes por lo que estaba siendo tratada con AINEs. En la exploración física destacaba una discreta ingurgitación yugular y una hepatomegalia de $3 \mathrm{~cm}$. En la radiografía de tórax se observaba cardiomegalia grado II. Ante la sospecha de dolor abdominal probablemente secundario a gastropatía por AINES e insuficiencia cardiaca no filiada ingresa en medicina interna para estudio.

La noche del ingreso se constata deterioro clínico progresivo con hipotensión, mala perfusión periférica, y signos de insuficiencia cardiaca derecha. El ECG mostraba taquicardia sinusal a $110 / \mathrm{min}$, microvoltaje generalizado y alternancia eléctrica. Se le realizó ecocardiagrama urgente que confirmó la sospecha de derrame pericárdico con taponamiento cardiaco. Se le practicó una pericardiocentesis evacuadora mejorando el cuadro clínico y permaneciendo estable durante varios días. En los controles rutinarios posteriores se constató la persistencia del derrame pericárdico y se realizó pericardiectomía con ventana pericárdica. El estudio del líquido pericárdico era compatible con exudado donde se aislaron células metastásicas de adenocarcinoma.

Con el juicio clínico de derrame pericardico metastásico se realizó un estudio de extensión con TAC toraco-abdominal donde destacaba le presencia de 
derrame pleural bilateral, múltiples adenopatias mediastínicas e imágenes líticas en columna dorsolumbar, así como mamografía bilateral que fue informada como normal y broncoscopia con lavado bronquial donde no se observaron alteraciones.

Existía elevación de los siguientes marcadores; CEA $33.62 \mathrm{ng} / \mathrm{ml}(0-4.7)$ Cifra 21: $14.92 \mathrm{ng} / \mathrm{ml}(0-$ 3) CA 15.3: $56.79 \mathrm{U} / \mathrm{ml}(0-31) \mathrm{CA} 19.9: 377.30$ $\mathrm{U} / \mathrm{ml}(0-40)$

Con el diagnóstico de tumor de origen desconocido de probable origen pulmonar, dado su localización, o mamario, dado el sexo del paciente y la alta prevalencia de este tumor, se decidió no realizar más pruebas diagnósticas y se inició tratamiento quimioterapico para tumores de origen desconocido con paclitaxel y carboplatino.

Una vez estabilizada la situación clínica la enferma recibió alta con ventana pericardica y drenaje pericardico.

A la semana del alta la enferma reingresó por empeoramiento clínico con signos de insuficiencia cardiaca derecha. Se realizó ecocardiograma donde se observaba taponamiento cardiaco por mal funcionamiento de la ventana pericárdica probablemente secundario a coágulos y fibrosis. Fue valorada por el servicio de cirugía cardiovascular que desestimó la intervención quirúrgica. La paciente fue existus dos días después.

\section{Discusión}

Los tumores de origen desconocido (TOD) representan un grupo heterogéneo de enfermedades neoplásicas que se presentan con metástasis sin la identificación del tumor primario. A pesar de los continuos avances en el diagnóstico y tratamiento, el pronóstico sigue siendo malo con una mediana de supervivencia de 6-10 meses.

Los pacientes con TOD son sometidos la mayoría de las veces a múltiples y costosos procedimientos terapéuticos la mayoría de ellos desagradables para el paciente y que no van a reflejar un aumento en la supervivencia del mismo 5 .

El taponamiento cardiaco como forma de presentación de un adenocarcinoma es muy poco frecuente, datando la serie más extensa de 13 pacientes de los cuales en 10 se identificó el tumor primario siendo en 7 un cáncer de pulmón, y en los restantes un cáncer gástrico, un cáncer de mama y un cáncer de tiroides ${ }^{6}$.

En nuestro caso la presencia de derrame pericárdico de metástasis de adenocarcinoma orientó nuestra sospecha diagnóstica hacia dos posibles neoplasias que pueden comportarse de este modo como son el cáncer de mama y el cáncer de pulmón. Se decidió administrar tratamiento quimioterápico con paclitaxel y carboplatino, dos agentes citotóxicos activos en ambos tumores ${ }^{7}$. A pesar de la administración de un primer ciclo el tumor progresó reproduciéndose el taponamiento.

El objetivo de este caso clínico es destacar la escasa justificación de una aplicación exhaustiva de todos los estudios diagnósticos. Las pruebas a realizar estarán en función del síndrome clínico y de los síntomas y signos que presente el paciente . $^{8}$

Nuestro esfuerzo debe dirigirse a las áreas donde la probabilidad de encontrar al tumor primario sea mayor ${ }^{9}$, en nuestro caso el pulmón y la mama. Sin olvidar que la prueba que más información aporta es el estudio histológico, realizando una nueva biopsia o estudiando junto con el patólogo la ya existente.

Debemos centrarnos en identificar aquellos tumores con posible curación como son los tumores de células germinales, la enfermedad de Hodking, los Linfomas no Hodking y los tumores trofoblásticos, así como aquellos donde la quimioterapia es altamente efectiva como en el carcinoma de mama, de ovario y en el carcinoma microcítico de pulmón. Un grupo especial lo constituyen aquellos tumores donde existe un tratamiento hormonal no tóxico como son el cáncer de mama, de próstata y de endometrio $^{10}$.

No está justificada la aplicación exhaustiva de toda la batería de pruebas diagnósticas de que disponemos, pero es fundamental diagnosticar aquellos tumores potencialmente curables.

Correspondencia:

Dr. D. Soto de Prado Otero Servicio de Oncología Médica Hospital Clínico Universitario $\mathrm{P}^{\mathrm{o}}$ San Vicente, 58-182 E-37007 Salamanca E-mail: sotodeprado@yahoo.es albertocana@yahoo.es 


\section{Bibliografía}

1. Greco FA, Hainsworth JD. Tumors of unknown origin. Ca Cancer J Clin 1992; 42:96-115.

2. Nystrom JS, Weiner JM, Wolf RM, et al. Identifying the primary site in metastatic cancer of unknown origin. JAMA 1979; 241:381-383.

3. Milliken ST, Tattersall MH, Woods RL, et al. Eur J Cancer Clin Oncol 1987; 23: 1645-1648.

4. Greco FA, Burris HA, Erland JB, et al. Carcinoma of unknown primary site. Cancer 2000; 89; 2655-2660.

5. Schapira DV, Levine RB. The need to consider survival, outcome, and expense when evaluating and treating patients with unknown primary carcinoma. Arch Intern Med 1995; 155:2050-2054

6. De la Gándara I, Espinosa E, Gómez Cerezo J, et al. Pericardial tamponade as the first manifestation of adenocarcinoma. Acta Oncol 1997; 36:429-31.
7. Hainsworth JD, Erland JB, Kalman LA, et al. Carcinoma of unknown primary site. Treatment with 1 hour paclitaxel, carboplatin and extended-schedule etoposide. J Clin Oncol 1997; 15: 2385-2393.

8. Robert G. Clinical evaluation of the patient with an unknown primary tumor. American Society of clinical oncology. Educational Book 2002; 327-334.

9. Abbruzzese JL, Abbruzzese MC, Lenzi R, et al. Analysis of a diagnostic strategy for patients with suspected tumors of unknown origin. J Clin Oncol 1995; 13: 20942103.

10. Frank A. Cancer of unknown primary site. Cancer: Principles and practice of Oncology, 6th Edition, edited by Vincent T. De Vita, Jr Samuel Hellman, Steven A. Rosenberg. Lippincott-Raven Publishers, Philadelphia 2001; pp 2537 2559. 